\title{
Interactive comment on "Variation in altitude of high-frequency enhanced plasma line by the pump near the 5th electron gyro-harmonic" by Jun Wu et al.
}

Jun Wu et al.

wujun1969@163.com

Received and published: 14 April 2019

Due to many Figures and functions, we would like to submit the reply for reviewer in a Supplement document. We are trying to express that this paper should be new and meaningful. Indeed, The descents of the HFPL and HFIL altitudes at EISCAT UHF, VHF and MUIR were frequently observed, which were attributed to the change in the profile of electron density [Djuth et al. 1994; Kosch et al., 2004; Dhillon et al., 2005; Ashrafi et al., 2006; 2007] or the artificial descending layers [Streltsov et al., 2018]. In this paper, however, we suggested an alternative explanation for the descents of the HFPL, namely, the descents of the HFPL may be due to the enhanced 
electron temperature on the traveling path of the enhanced Langmuir wave rather than the change in the profile of electron density. We sincerely request the reviewer to re-consider the comment and conclusion please. If so, we will make some modification and clarity.

Please also note the supplement to this comment:

https://www.ann-geophys-discuss.net/angeo-2019-23/angeo-2019-23-AC2supplement.pdf

Interactive comment on Ann. Geophys. Discuss., https://doi.org/10.5194/angeo-2019-23, 2019. 